Digitalisierung und Vernetzung der Industrieproduktion

\section{Nachhaltigkeitsaspekte von Industrie 4.0}

\author{
Aus Sicht der Nachhaltigkeitsforschung sind die möglichen \\ Veränderungen industrieller Produktion von großem \\ Interesse. Welche Potenziale ergeben sich durch Industrie 4.0 \\ für eine nachhaltigere Art des Produzierens? \\ Von Grischa Beier, Silke Niehoff und Achim Maas
}

W as steckt hinter dem Schlagwort Industrie 4.0? Fakt ist, dass derzeit vor allem in Europa, den USA und China verstärkt zur Digitalisierung der Industrieproduktion geforscht wird. Die Industrie erwirtschaftet in Deutschland etwa ein Drittel der Brutto-Wertschöpfung (Eurostat 2015) und stellt knapp $20 \%$ aller Arbeitsplätze (Eurostat 2014a). Gleichzeitig konsumiert die Industrie fast 30\% der Endenergie (Eurostat 2014b) und ist mit 19\% einer der größten Treibhausgasemittenten in Deutschland (Eurostat 2014a). Sie tangiert somit alle drei Dimensionen der Nachhaltigkeit. Dieser Artikel beleuchtet mögliche Implikationen von Industrie 4.0 für die Nachhaltigkeitsdebatte.

\section{Was bedeutet Industrie 4.0?}

Industrie 4.0 bezeichnet die zunehmende Verschmelzung der physischen Welt der industriellen Produktion mit der digitalen Welt der Informationstechnik. Diese ermöglicht eine digitale Vernetzung unterschiedlicher, bisher häufig getrennter Produktionssysteme. Im Kontext industrieller Produktion müssen dazu Bauteile und Maschinenkomponenten eindeutig identifizierbar und mit einer Datenverbindung ausgestattet sein, damit sie in der Lage sind, miteinander $\mathrm{zu}$ kommunizieren und Daten auszutauschen. Sie sollten zudem über Sensoren sowie ggf. Aktoren verfügen, um relevante Informationen erfassen und bei Bedarf Aktionen einleiten zu können. Ein Hauptziel von Industrie
4.0 ist dabei, die Produktions- und Logistikprozesse zu flexibilisieren, sodass kundenspezifische Wünsche einfacher und schneller realisierbar werden (Forschungsunion 2013).

\section{Nachhaltigkeitsaspekte}

Die Digitalisierung und Vernetzung industrieller Produktion findet heute bereits statt und wird in den nächsten Jahren weiter voranschreiten. Unklar ist hingegen, ob und wie einzelne Nachhaltigkeitsparameter im Zuge von Industrie 4.0 beeinflusst werden. Häufig wird ein $\mathrm{Zu}$ wachs des Bruttoinlandsproduktes (1\% pro Jahr) sowie an Arbeitsplätzen (plus 390.000) und eine gesteigerte Effizienz (18\% in fünf Jahren) prognostiziert (The Boston Consulting Group 2015; PricewaterhouseCoopers AG 2014). Wissenschaftlich fundierte Untersuchungen sind derzeit aber noch rar. So zeichnet die Studie von Frey und Osborne (2013) ein deutlich skeptischeres Zukunftsbild, nach dem bis zu 47\% der Arbeitsplätze in den USA durch Computerisierung bedroht seien. Eine Adaption dieser Untersuchungsmethode auf den europäischen Kontext kommt für Deutschland auf einen Wert von 51\% (Bowles 2014).

Das Institute for Advanced Sustainability Studies (IASS) führt das Projekt „Nachhaltigkeitsaspekte von Industrie 4.0“ durch, um zu ermitteln, wie ausgewählte Nachhaltigkeitsparameter durch Industrie 4.0 beeinflusst werden (siehe Abbildung 1). Neben dem Themenfeld Arbeit, das in den Diskursen bereits prä- sent ist, widmet sich das Projekt einer wissenschaftlichen Betrachtung der vielfach postulierten Steigerung an Ressourceneffizienz sowie möglichen Synergieeffekten mit der Energiewende.

\section{Projektergebnisse}

2014 veranstaltete das IASS eine Reihe von Arbeitsgesprächen mit Akteuren aus Politik, Wirtschaft und Gewerkschaften, um die unterschiedlichen Vorstellungen und Interessenlagen zu diskutieren. Anschließend wurde eine vorwiegend an Unternehmen gerichtete Online-Befragung durchgeführt. Insgesamt nahmen 102 Personen an der Befragung teil.

Dabei gehen über $90 \%$ der Befragten davon aus, dass zukünftig der Anteil von Informations- und Kommunikationstechnologie an Produkten zunimmt [1]. Dies muss in deren Rohstoffbilanzierung mit einbezogen werden. Gleichzeitig ist die Ressourceneffizienz für $92 \%$ der Befragten im Rahmen von künftigen, strategischen Unternehmensentscheidungen wichtig oder sehr wichtig.

Eine flexiblere Produktion ermöglicht, verstärkt zu Zeiten zu produzieren, in denen viel elektrische Energie von fluktuierenden erneuerbaren Energiequellen ins Netz gespeist wird. Rund $41 \%$ der Befragten können sich vorstellen, dass ihr Unternehmen diese gewonnene Flexibilität in der Produktion nutzt (siehe Abbildung 2, links). Rund ein Drittel der Befragten gaben zudem an, dass ihr Unternehmen in Zukunft selbst Energie erzeugt (siehe Abbildung 2, rechts).

Die verstärkte digitale Vernetzung bezieht nicht nur die Produktionsmittel innerhalb eines Unternehmens mit ein, sondern hat auch Auswirkungen auf die Zulieferkette. $72 \%$ der Befragten gaben an, dass einzelne Komponenten zunehmend zugeliefert werden. Diese Dezentralisierung von Produktion in Zulieferernetzwerke beeinflusst die Transportwege und somit potenziell auch dadurch anfallende Ressourcenverbräuche und Emissionen.

Wie könnte ein hypothetisches Zukunftsszenario aussehen, das die Nachhaltigkeitspotenziale und Industrie 4.0 


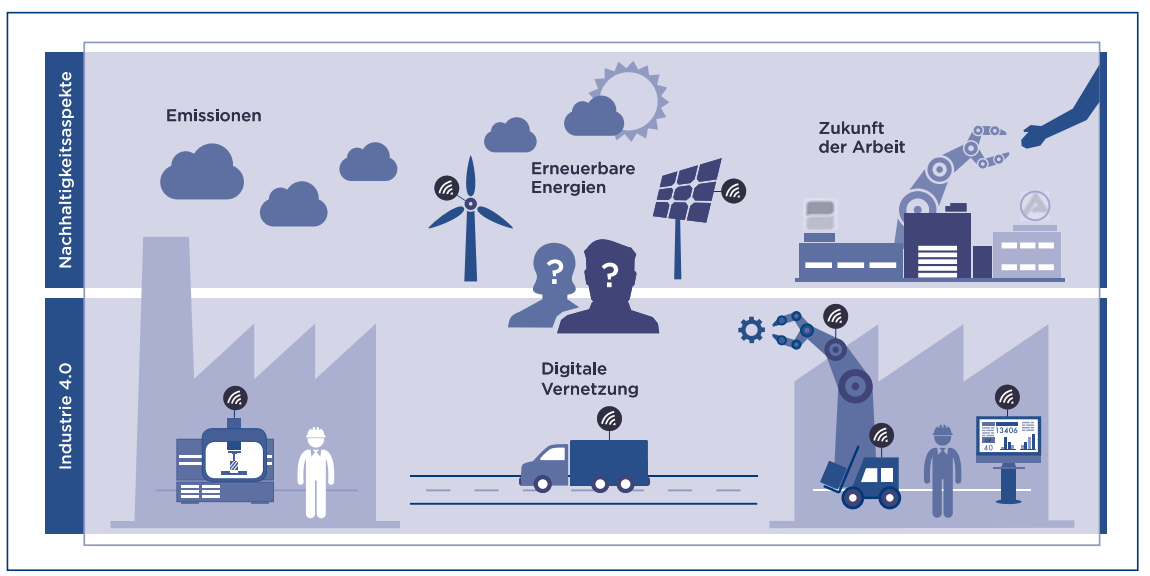

Abbildung 1: Schematische Darstellung möglicher Nachhaltigkeitsaspekte von Industrie 4.0

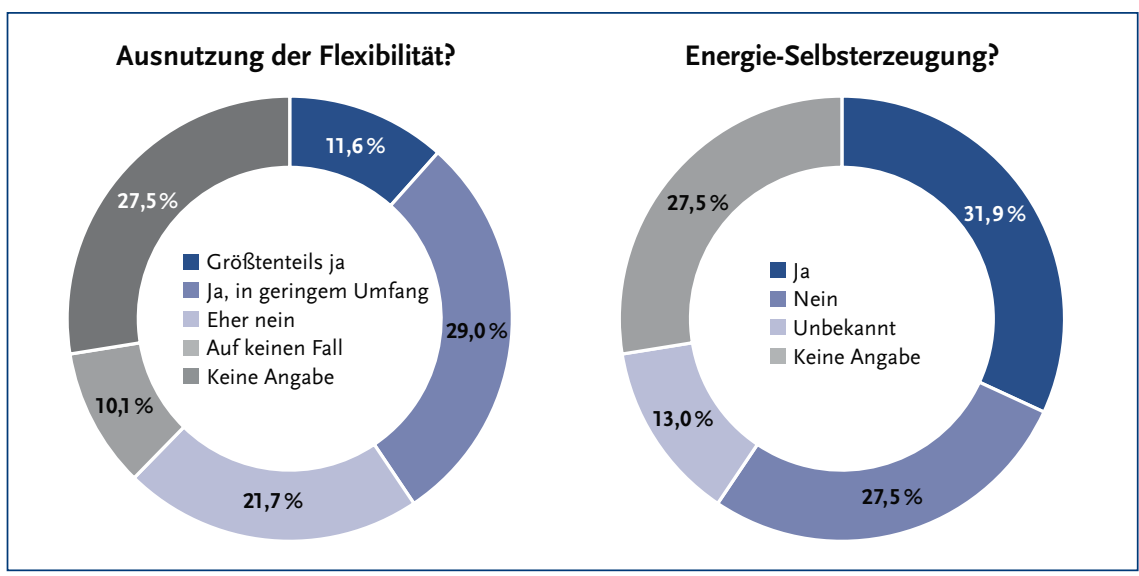

Abbildung 2: Umfrageergebnisse zur Ausnutzung der Produktionsflexibilität zum Bezug preiswerten Stroms (links) und Plänen zur eigenen Energieerzeugung (rechts)

miteinander verknüpft? Einen ersten Eindruck, der im Laufe des Projekts weiter verfeinert werden soll, kann das folgende Szenario „Unterstützung der Energiewende durch Industrie 4.0" vermitteln.

\section{Ausblick}

Die Einzelfertigung in der Fabrik der Zukunft ist durch die weitgehende Entkoppelung der Steuerungsprozesse von menschlichem Einfluss zeitlich hochgradig flexibel. Energieintensive Fertigungsprozesse erfolgen, wenn regenerative Energie im Überangebot vorhanden ist. Die nun algorithmisch prognostizierbaren flexiblen Lasten produzierender Unternehmen sind zudem Teil von virtuellen Kraftwerken. Hierdurch können diese Unternehmen zusätzliche Er- löse generieren. Dies ist zum Beispiel im Regelleistungsmarkt, um Schwankungen im Stromnetz auszugleichen, möglich. Ferner ist es Unternehmen nun erlaubt, überschüssige Prozess-Abwärme, die also nicht für interne Verfahren oder zur Kühlung benötigt wird, für die umliegenden privaten Verbraucher flexibel ins Wärmenetz einzuspeisen.

$\mathrm{Ob}$ die Entwicklung in die geschilderte Richtung gehen wird, ist offen. Das Gegenteil, dass die Digitalisierung selbst einen höheren Rohstoffverbrauch fordert als durch effizientere Produktion eingespart werden kann, ist genauso vorstellbar. Weitgehend unbestritten ist hingegen, dass Digitalisierung und Vernetzung in den hoch entwickelten Industrien verstärkt Einzug halten werden. Ziel und Anspruch sollte es daher sein, das technologisch Mögliche so mit ge- eigneten Rahmenbedingungen (legislativ, fiskalisch, infrastrukturell) zu begleiten, dass Industrie 4.0 perspektivisch zu nachhaltigerem Wirtschaften führt.

\section{Anmerkung}

[1] Die Beantwortung jeder Frage war fakultativ. Alle Prozentsätze beziehen sich daher auf die Anzahl der jeweils Antwortenden.

\section{Literatur}

Bowles, J. (2014): The computerisation of European jobs. Who will win and who will lose from the impact of new technology onto old areas of employment?

Eurostat (2014a): National Accounts by 10 branches - employment data. Basisjahr 2012 Im Internet unter: http://appsso.eurostat. ec.europa.eu/nui/show.do?dataset=nama nace10_e\&lang=en

Eurostat (2014b): Final energy consumption by sector. Basisjahr 2012. Im Internet unter: http://ec.europa.eu/eurostat/tgm/table.do? tab=table\&init=1\&language $=$ en \&pcode $=$ tsdpc320\&plugin $=1$

Eurostat (2015): National Accounts by 10 branches - aggregates at current prices. Basisjahr 2012. Im Internet unter: http:// appsso.eurostat.ec.europa.eu/nui/show. do?dataset=nama_nace10_c\&lang=en

Frey, C. B./Osborne, M. A. (2013): The future of employment: how susceptible are jobs to computerisation? OMS Working Paper.

PricewaterhouseCoopers AG (2014): Industrie 4.0 - Chancen und Herausforderungen der vierten industriellen Revolution.

The Boston Consulting Group (2015): Industry 4.0. The Future of Productivity and Growth in Manufacturing Industries.

Forschungsunion Wirtschaft - Wissenschaft/ Deutsche Akademie der Technikwissenschaften (2013): Umsetzungsempfehlungen für das Zukunftsprojekt Industrie 4.0.

AUTOREN + KONTAKT

Dr. Grischa Beier, Silke Niehoff und Achim Maas sind Wissenschaftler am IASS Potsdam.

IASS, Institute for Advanced

Sustainability Studies e. V., Berliner Straße 130, 14467 Potsdam. Tel.: +49 331 28822-342, E-Mail: grischa.beier@iass-potsdam.de, Internet: www.iass-potsdam.de 